\title{
Subfoveal Choroidal Thickness in Relation to Age and Refractive Error
}

\author{
SAFAA GALAL, M.Sc.; MOHAMMAD M. KHAFAGY, M.D.; HEBA M. AHMED, M.D. and \\ SOHEIR M. ESMAT, M.D.
}

The Department of Ophthalmology, Faculty of Medicine, Cairo University

\begin{abstract}
Background: The choroid is a highly vascular structure and it undergoes structural and functional alterations with increasing age which may affect the visual function. In addition, choroidal thickness differs according to the state of refraction and axial length.
\end{abstract}

Aim of the Study: To correlate between the subfoveal choroidal thickness and various clinical data including age, error of refraction, and best corrected visual acuity.

Patients and Methods: This cross sectional, nonrandomized, non-controlled study was conducted on 124 eyes. Measurement of the subfoveal choroidal thickness was done using spectral domain optical coherence tomography in the Investigative and Laser Unit of the Ophthalmology Department of Kasr Al-Ainy Hospital.

Results: Results showed that thinner choroid was associated with older age $(r=-0.264, p$-value $=0.003)$ and myopic refraction $(r=0.243, p$-value $=0.007)$, while thicker choroid was related to a better visual acuity $(r=0.199, p$-value $=0.026)$ Also, there was no significant difference in choroidal thickness measurements by the two obsevers $(p$-value $=0.161)$.

Conclusion: Older persons and eyes with myopic refraction have a thinner choroid. A thicker choroid is related to a better visual acuity. No inter-observer variation was found regarding choroidal thickness measurements.

Key Words: Subfoveal choroidal thickness - Age - Refractive error.

\section{Introduction}

THE choroid is a highly vascular structure and similar to any microvasculature in the body, it is likely to undergo structural and functional alterations with increasing age. This generally involves a reduction in perfusion to the outer retina, which may have an impact on visual function [1] .

Correspondence to: Dr. Safaa Galal, The Department of Ophthalmology, Faculty of Medicine, Cairo University
According to histological studies, the human subfoveal Choroidal Thickness (CT) is approximately $200 \mathrm{gm}$ thick at birth and decreases to about $80 \mathrm{gm}$ by age 90 [2] with the choroid being thinner in eyes with longer axial length [3].

The Beijing Eye Study reviewed subfoveal CT in 3,233 subjects and found a number of associations [4]. Multivariate analysis found that subfoveal CT increased significantly with younger age, shorter axial length, deeper anterior chamber depth, thicker lens, and flatter corneas [4]. However, CT was not significantly associated with blood pressure, ocular perfusion pressure, intraocular pressure, cigarette smoking, alcohol consumption, serum concentrations of lipids and glucose, diabetes mellitus, and arterial hypertension [4].

Adequate visualization of the choroid using Spectral Domain Optical Coherence Tomography (SD-OCT) has not been possible until recently, due to its posterior location and the presence of pigmented cells that attenuate the incident light. In 2008, Spaide et al., [5] were the first to visualize the choroid by inverting SD-OCT images, SDOCT images are highest in resolution near the zero-delay line. Thus, when the image is inverted, even if some resolution is lost at the vitreous and the retina, the resolution at the choroid is excellent [5].

Branchini et al., [6] investigated the reproducibility of choroidal thickness measurements in normal subjects on three Spectral Domain Optical Coherence Tomography (SD-OCT) instruments, Heidelberg Spectralis, Zeiss Cirrus HD-OCT (Carl Zeiss MeditecInc, Dublin, CA, USA), and OptovueRTVue (Optovue Inc., Fremont, CA, USA). They found that in their population of young healthy adults with normal vision, there was good repro- 
ducibility between choroidal thickness measurements of images acquired with Cirrus, Spectralis and RTVue. In addition, there was good interobserver correlation for all choroidal thickness measurements [6]

\section{Patients and Methods}

This cross sectional, observational, nonrandomized, non-controlled study was conducted on 124 eyes in the Investigative and Laser Unit of the Ophthalmology Department of Kasr Al-Ainy Hospital during the year 2016.

The involved persons were more than 40 years of age with no prior history of retinal diseases including diabetic retinopathy, age-related macular degeneration, or retinal vein occlusion. We excluded any media opacity affecting the OCT image quality. We also excluded high myopia ( $>6 \mathrm{D})$ and history of ocular inflammation, trauma, or surgery.

All participants received a thorough explanation of the study design and aims. Study participants gave informed consent before initiation of any study-related procedures, and the study was conducted in compliance with informed consent regulations. All patients were subjected to a full ophthalmological examination, including full medical and ophthalmic history taking, assessment of Best Corrected Visual Acuity (BCVA) using the decimal system, external examination, slit lamp examination, assessment of the intraocular pressure using Goldmann applanation tonometry, and dilated fundus examination using slit lamp biomiocroscopy with a +90D lens and the indirect ophthalmoscope with a +20D lens. Measurement of the subfoveal choroidal thickness was done using SD-OCT: Optovue RTVue 100 (Optovue Inc., Fremont, CA, USA). The choroidal thickness was measured manually at the subfovea starting from the outer portion of the hyperreflective line corresponding to the RPE to the inner surface of the sclera, using the line segment measurement tool Fig. (1). The choroid was measured by two independent graders then their measurements were averaged.

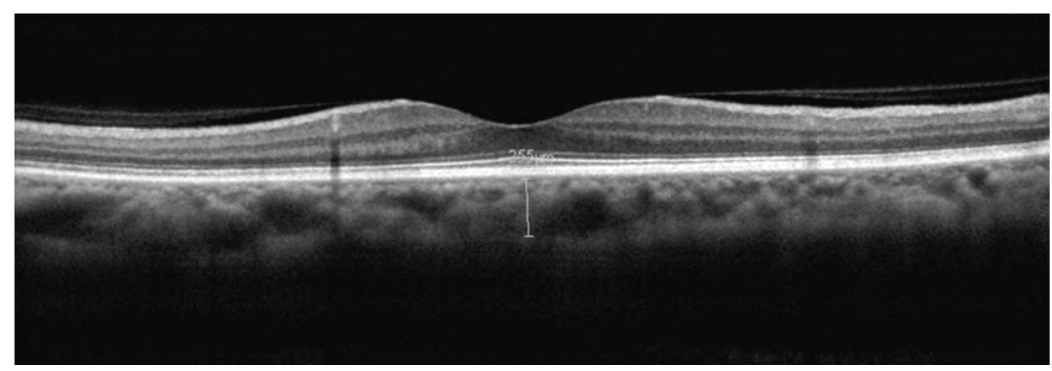

Fig. (1): Subfoveal choroidal thickness of one of our patients=255

\section{Results}

124 eyes were included in the study, $41.9 \%$ were males and $58.1 \%$ were females. The mean age was $55.5 \pm$ years, SD of \pm .6 years (range: 45 75 years). The mean BCVA was 0.4, SD of \pm 2 (range: 0.05-1). The mean spherical equivalent was -0.7 diopters, SD of $\pm 2.7 \mathrm{D}$ (Range: $-6 \mathrm{D}$ to $+6 \mathrm{D}$ ). The mean subfoveal choroidal thickness was 232 s SR of \pm 52 F(range: $114-397$

Our study demonstrated a negative correlation between subfoveal choroidal thickness and age ( $r=$ $-0.264, p$-value $=0.003$ ), while there was a positive correlation between subfoveal choroidal thickness and each of spherical equivalent $(r=0.243, p$-value $=0.007)$ and BCVA $(r=0.199, p$-value $=0.026)$.

We also compared the subfoveal choroidal thickness measurements of the two observers to detect any inter-personal variation in the measurement of the subfoveal choroidal thickness.
Table (1): Measurements of the two observers

\begin{tabular}{lll}
\hline & Mean $\begin{array}{c}\text { Std. } \\
\text { deviation }\end{array}$ \\
\hline Choroidal thickness by the first observer & 234 & 57 \\
Choroidal thickness by the second observer & 230 & 52 \\
\hline
\end{tabular}

The mean of the first observer measurements was 234 Sreof 57 while the mean of the second observer measurements was 230 SS of 52 There was a positive correlation between the subfoveal choroidal thickness measured by the two observers $(r=0.799, p$-value $<0.001)$, with no significant difference between the two $(p$-value $=$ $0.161)$.

\section{Discussion}

The present study showed a significant negative correlation between subfoveal choroidal thickness and age $(r=-0.264, p$-value $=0.003)$. This is similar 
to previous studies which demonstrated that older persons and eyes with longer axial length or myopic refraction have a thinner choroid [3,7].

Our study also demonstrated a significant positive correlation between subfoveal choroidal thickness and spherical equivalent ( $r=0.243, p$-value $=$ $0.007)$, reflecting increased choroidal thickness in hypermetropia and decreased choroidal thickness in myopia. This is similar to the results obtained by Abbey et al., [8] who found a negative correlation between axial length and choroidal thickness. They also found a strong negative correlation between choroidal thickness and age.

The current study demonstrated a significant positive correlation between subfoveal choroidal thickness and BCVA ( $r=0.199, p$-value $=0.026)$. This may suggest a better choroidal blood supply to the macula with thicker choroid which is reflected in terms of better BCVA. This may be similar to the results obtained by Ho et al., [9] and FloresMoreno [10], but they studied highly myopic eyes. They found that choroidal thickness decreased with age and severity of myopia, while visual acuity decreased in line with decreasing subfoveal choroidal thickness. A reduction in choroidal thickness was related to aging and the severity of myopia, whereas visual acuity depended on subfoveal choroidal thickness $[\mathbf{9 , 1 0}]$

The current study showed a significant positive correlation between the subfoveal choroidal thickness measured by the two observers $(r=0.799, p$ value $<0.001)$, with no significant difference between the two $(p$-value $=0.161)$. This is similar to the results by Branchini et al., [6], where there was good inter-observer correlation for all choroidal thickness measurements.

\section{Conclusion:}

Thinner choroid was found in older persons and eyes with myopic refraction. A thicker choroid is related to a better visual acuity. No inter-observer difference was found regarding choroidal thickness measurements.

\section{References}

1- LAVIERS H. and ZAMBARAKJI H.: Enhanced depth imaging-OCT of the choroid: A review of the current literature. Graefes Arch. Clin. Exp. Ophthalmol., 252: 1871-83, 2014.

2- RAMRATTAN R.S., VAN DER SCHAFT T.L., MOOY C.M., et al.: Morphometric analysis of Bruch's membrane, the choriocapillaris and the choroid in aging. Invest. Ophthalmol. Vis. Sci., 35: 2857-64, 1994.

3- ESMAEELPOUR M., POVAZAY B., HERMANN B., et al.: Three dimensional 1060-nm OCT: Choroidal thickness maps in normal subjects and improved posterior segment visualization in cataract patients. Invest. Ophthalmol. Vis. Sci., 51: 5260-6, 2010.

4- WEI W., XU L., JONAS J., et al.: Subfoveal choroidal thickness: The Beijing eye study. Ophthalmology, 120 (1): 175-80, 2012.

5- SPAIDE R.F., KOIZUMI H. and POZZONI M.C.: Enhanced depth imaging spectral-domain optical coherence tomography. Am. J. Ophthalmol., 146 (4): 496-500, 2008.

6- BRANCHINI L., REGATIERI C.V., FLORES-MORENO I., et al.: Reproducibility of Choroidal Thickness Measurements acrossThree Spectral Domain Optical Coherence Tomography Systems. Ophthalmology, 119 (1): 119-23, 2012.

7- IKUNO Y., KAWAGUCHI K., NOUCHI T., et al.: Choroidal thickness in healthy japanese subjects. Investigative ophthalmology \& visual science, 51: 2173-6, 2010.

8- ABBEY A.M., KURIYAN A.E., MODI Y.S., et al.: Optical coherence tomography measurements of choroidal thickness in healthy eyes: Correlation with age and axial length. Ophthalmic Surg Lasers Imaging Retina, 46 (1): 18-24, 2015. doi: 10.3928/23258160-20150101-03.

9- HO M., LIU D.T., CHAN V.C., et al.: Choroidal thickness measurement in myopic eyes by enhanced depth optical coherence tomography. Ophthalmology, 120 (9): 1909 14, 2013.

10- FLORES-MORENO I., RUIZ-MEDRANO J., DUKER J.S. and RUIZ-MORENO J.M.: The relationship between retinal and choroidal thickness and visual acuity in highly myopic eyes. Br. J. Ophthalmol., 97 (8): 1010-3, 2013.

\section{علاقة سمك المشيمة تحت ماقولة العين بالسن والحالة الإنكسارية للعين}

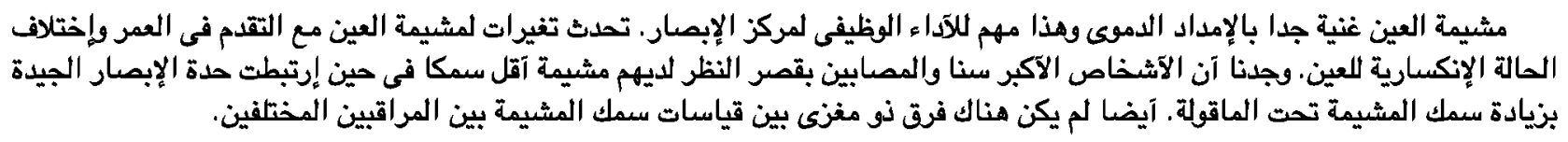

